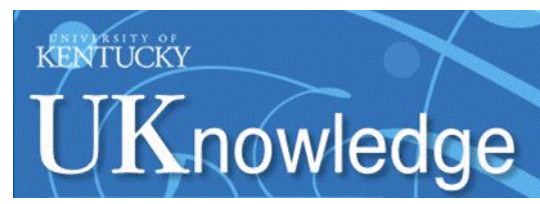

University of Kentucky

UKnowledge

$12-2014$

\title{
Focusing on Drug Versus Disease Mechanisms and on Clinical Subgrouping to Advance Personalised Medicine in Psychiatry
}

Jose de Leon

University of Kentucky, jdeleon@uky.edu

Follow this and additional works at: https://uknowledge.uky.edu/psychiatry_facpub

Part of the Psychiatry and Psychology Commons

Right click to open a feedback form in a new tab to let us know how this document benefits you.

\section{Repository Citation}

de Leon, Jose, "Focusing on Drug Versus Disease Mechanisms and on Clinical Subgrouping to Advance Personalised Medicine in Psychiatry" (2014). Psychiatry Faculty Publications. 29.

https://uknowledge.uky.edu/psychiatry_facpub/29

This Article is brought to you for free and open access by the Psychiatry at UKnowledge. It has been accepted for inclusion in Psychiatry Faculty Publications by an authorized administrator of UKnowledge. For more information, please contact UKnowledge@lsv.uky.edu. 
Focusing on Drug Versus Disease Mechanisms and on Clinical Subgrouping to Advance Personalised Medicine in Psychiatry

Digital Object Identifier (DOI)

http://dx.doi.org/10.1017/neu.2014.14

Notes/Citation Information

Published in Acta Neuropsychiatrica, v. 26, issue 6, p. 327-333.

(C) Scandinavian College of Neuropsychopharmacology 2014

The copyright holder has granted the permission for posting the article here.

The document available for download is the authors' post-peer-review final draft of the article. 
Words in text: 3186

Tables: 0

Figures: 0

\section{PERSPECTIVE:}

Focusing on drug versus disease mechanisms and on clinical subgrouping to advance personalized medicine in psychiatry

Jose de Leon, M.D.*

*University of Kentucky Mental Health Research Center at Eastern State Hospital, Lexington, KY, and Psychiatry and Neurosciences Research Group (CTS-549), Institute of Neurosciences, University of Granada, Granada, Spain.

Running title: Personalized medicine psychiatry Corresponding author: Jose de Leon, M.D., UK Mental Health Research Center at Eastern State Hospital, 1350 Bull Lea Road, Lexington, KY 40511. Phone (859) 246-8440. Fax (859) 246-8446. e-mail: jdeleon@uky.edu 


\begin{abstract}
Personalized medicine has finally been featured in psychiatric journals but psychiatrists have mainly focused on the promise of using disease mechanisms to personalize treatment. Psychiatric disorders such as schizophrenia and depression are not diseases, in the medical sense, and are probably more like syndromes. Instead of spending much time and effort focusing on the mechanisms of diseases that may instead be syndromes, the author believes that psychiatrists should 1) learn more about personalizing prescription via drug mechanisms, a pharmacological approach to personalized medicine; and 2) reconsider prior attempts by traditional clinical psychopharmacologists to use sophisticated clinical approaches that try to subdivide psychiatric syndromes into groups that may be more homogenous for treatment response.
\end{abstract}

Key words: Alzheimer disease;individualized medicine; personalized medicine; personalized prescription; pharmacogenetics; pharmacogenomics; psychiatry; psychopharmacology; psychotropic drugs.

\title{
Summations:
}

-Personalized medicine has finally arrived in psychiatric journals but these journal articles have mainly focused on the promise of using disease mechanisms to personalize treatment.

\section{Perspectives:}

-Psychiatric disorders such as schizophrenia and depression are not diseases, in the medical sense, and are probably more like syndromes. Focusing on their disease mechanism does not appear to be a good idea.

-Psychiatrists should learn more about personalizing prescription via drug mechanisms, a pharmacological approach to personalized medicine. 
-Psychiatrists need to reconsider prior attempts by traditional clinical psychopharmacologists to use sophisticated clinical approaches that try to subdivide psychiatric syndromes into groups that may be more homogenous for treatment response. 
Personalized medicine has been a fashionable concept that has been endorsed by pharmacological journals for almost 20 years, but has not reached psychiatric journals until more recently. The simple idea behind this concept is that each patient is different and therefore each individual needs to be treated differently. Everybody seems to agree that each patient is different but there is disagreement on how to best apply this in practice; the recommendations for implementation vary according to the background and interest of each author using the term "personalized medicine". This is best illustrated by the first psychiatric book (1) using this term, written in 1952 by Osborn, a psychiatrist with a psychoanalytic approach who used it to explain that psychiatrists should treat each patient as a unique individual according to his/her unique psychological mechanisms. Obviously, the current approaches to personalized medicine in psychiatry are not related to psychoanalytic theory, but the emphasis on uniqueness is shared.

This perspective article discusses how personalized medicine can be used in psychiatry, including the approaches 1) in pharmacological journals over the last 20 years, 2) in recent psychiatric journals, and 3) in traditional clinical psychopharmacology. First, the tradition of personalized medicine in pharmacology was developed in the context of pharmacogenetics and drug mechanisms (differences in drug response based on differences in drug mechanisms). Second, psychiatric journals have recently referred to personalized medicine in the context of the promise of biomarkers which could explain disease mechanisms in psychiatry (differences in drug response based on differences in disease mechanisms). Third, before DSM-III nosology was developed, psychopharmacologists traditionally classified patients in subgroups based on clinical symptoms which may influence treatment response (differences in drug response based on differences in clinical presentation within a psychiatric syndrome such as schizophrenia).

\section{Personalized medicine and drug mechanisms}


The history of the concept of personalized medicine within pharmacology has been reviewed in a prior article (2); therefore, it is only briefly summarized and updated here. At the beginning of the 20th century, "chemical individuality" in drug response was hypothesized (3), but it was not demonstrated until the 1950s when pharmacologists observed that some severe adverse drug reactions (ADRs) only occurred in a small number of patients who were thought to have peculiar genetic profiles. This led to the term "pharmacogenetics" (4). The Second World War provided an impetus to drug development and the development of pharmacological laboratories for better understanding drug response (5). For the next 20-30 years these laboratories made progress in describing the pharmacokinetic and pharmacodynamic mechanisms behind drug response (6). In the 1980s-90s the various genes associated with the cytochrome P450 (CYP) isoenzymes were discovered, along with their genetic variations (7). In the late 1990s, advances in genetics which allowed parallel genetic testing (testing for multiple genes at the same time) by using the so-called DNA microarrays (8) led to pharmacogenomics, the idea that many genes may influence drug response. The ability to test many genes led, in 1997, to Science defining “personalized prescription" as "tailoring drugs to a patient's genetic makeup" and to the prediction that personalized prescription would "soon" reach clinical practice (9). The media, captivated by the race for the human genome, popularized the concepts of personalized medicine and personalized prescription. After the race was over, it became clear that factors other than genetics may be very important in drug response. Therefore, in the view of the author (10), personalized prescription can be expressed as personalizing dosing and/or drug selection and should include genetic as well as environmental (e.g., co-medications, herbal medicines, smoking, foods, beverages) and personal (e.g., age, gender, ethnicity, illness) variables in order to improve the safety and efficacy of psychopharmacological drugs. Moreover, 
psychiatry is increasingly combining new pharmacogenomic findings with therapeutic drug monitoring (11), particularly to guide personalized dosing (12). In the opinion of the author, the literature has not sufficiently recognized that each drug has its own pharmacokinetic and pharmacodynamic mechanisms (13); therefore, "each drug is an individual."

\section{Personalized medicine and disease mechanisms}

Further development of DNA microarray technology has allowed the testing of multiple other products besides DNA, including proteins, RNAs, and lipids, and led to the development of new diagnostic branches such as "protenomics", "transcriptomics" and "metabonomics". All of these new techniques have resulted in a new concept in drug development, namely, the biomarker. There are many definitions of biomarker, but Wagner proposed "a characteristic that is objectively measured and evaluated as an indicator of normal biological processes, pathogenic processes, or pharmacological response(s) to a therapeutic intervention" (14). There are different types of biomarkers, including the pharmacogenomic biomarkers described above as the pharmacokinetic and pharmacodynamic mechanisms behind drug response.

There has been a research boom in biomarkers in medicine both for practical and theoretical reasons. From the practical point of view, biomarkers have been particularly successful in oncology (15) for two reasons: 1) the cancerous tissue is available for study of the pathogenetic mechanisms, and 2) our understanding of these pathogenetic mechanisms is sufficiently developed to allow us to start selecting drugs according to the mechanisms in each individual patient. From the theoretical point of view, there has been a revolution in bioinformatics, including the development of a new scientific concept called "complexity" (16), reflecting the complexity of scientific exploration when there are multiple influences causing a phenomenon and the need exists for complex computer models to integrate all that is known 
about it. Complexity theory and its accompanying computer models are being introduced in medicine by using the so-called "network" medicine (17). Therefore, personalized medicine using disease mechanisms is becoming a fad in medicine.

If one is a psychiatrist who wants to think that psychiatry is not lagging behind other medical disciplines, one should endorse the idea of using disease mechanisms to personalize psychiatric treatment. As a matter of fact, many of the recent articles in psychiatric journals that focus on the future of psychiatry propose using disease mechanism to personalize psychiatric treatment, but these articles usually ignore the less glamorous drug response mechanisms proposed by pharmacologists. There are many of these articles in many different journals but the author can point out a few of them for the reader (18-22).

This author proposes that the path to personalized psychiatry which uses disease mechanisms while forgetting drug response mechanisms is the wrong road. He acknowledges that our knowledge of the use of pharmacokinetic and pharmacodynamic mechanisms for personalizing psychiatric treatment is pretty limited since it applies to a few drugs, and more fundamentally to pharmacokinetic mechanisms. Our understanding of pharmacodynamic mechanisms of psychiatric drugs is too limited to have much clinical relevance $(12,23,24)$. Personalized medicine based on drug mechanisms is a "humble" pathway not likely to lead to any major breakthroughs but could promote the use of currently available drugs in a better way.

It is not surprising that, if one wants to compete with cancer researchers in marketing and funding, one should propose that psychiatry can cure mental illnesses (25) by personalizing treatment (26), since oncology appears to be on the verge of curing many types of cancers. On the other hand, the author proposes that at the current time, despite the wide endorsement of psychiatric researchers of the disease mechanism to personalize psychiatry, it may be premature 
and, furthermore, a waste of time and money. This is based on two ideas: 1) psychiatry is lagging behind medicine and many of the psychiatric diseases, such as schizophrenia, are more syndromes than diseases; and 2) even if one focuses on diseases with known brain neuropathology, such as Alzheimer disease, the complexity of brain mechanisms currently appears insurmountable.

\section{The problem with the concept of disease in psychiatry}

In a JAMA article in 2005, McHugh (27) pointed out that psychiatry is 150 years behind medicine, so we are trying to do what $19^{\text {th }}$ century physicians did. At that time, there was a revolution characterized by the convergence of three ways of thinking, which have been called anatomoclinical (relating signs and symptoms with disturbances in specific organs), physiopathological (relating diseases or their symptoms/signs with disturbances in normal physiology) and etiopathological (finding specific causes of some diseases and their lesions) (28). This led to the revolutionary success of $20^{\text {th }}$ century medicine.

The problems with the scientific approach in psychiatry have been discussed previously by the author (29) but anyone with historical knowledge of psychiatric disease knows that diseases such as schizophrenia (30) are at best syndromes and not medical diseases in the traditional sense. Cancers, such as colon cancer, are being divided into different diseases based on pathogenic mechanism. Unfortunately, psychiatry is far from reaching that point. Moreover, we have no way of validating the diagnosis of most psychiatric disorders and establishing their boundaries with other psychiatric disorders.

\section{Problems dealing with brain complexity: Alzheimer disease}

There is no doubt that originally Alzheimer disease was a psychiatric disease. In 1907, Alzheimer, a psychiatrist, described the neuropathology in one case of presenile dementia with 
psychosis and in 1910 Kraepelin, a psychiatrist, (in the $8^{\text {th }}$ edition of his textbook) baptized it as a new illness (31). After including "senile" dementia cases within the boundaries of Alzheimer disease, a major development in neuroscience research occurred in 1990; it included genetic findings such as the association of Apolipoprotein E-4 with the age of onset of common lateonset Alzheimer disease.

It is now doubtful that Alzheimer disease can be called a psychiatric disease, since most of the new findings come from neurologists and other neuroscientists. To demonstrate, the author completed a PubMed search on 3/1/14 comparing articles from the last 10 years of two pairs of similar journals in neurology and in psychiatry. Neurology was compared with the American Journal of Psychiatry and JAMA Neurology (previously Archives of Neurology) with JAMA Psychiatry (previously Archives of General Psychiatry). Neurology published 912 articles on Alzheimer disease (or 8.2\%, 912/11156) versus 54 (or 1.7\%, 54/3428) in the American Journal of Psychiatry. JAMA Neurology published 501 articles (or 16.8\%, 501/2985) versus 48 (or 3.0\%, 48/1560) in JAMA Psychiatry. In both absolute and relative terms, Alzheimer disease is a major topic in neurological journals and a rather minor topic in psychiatric journals.

Let's stretch reality and consider Alzheimer disease a psychiatric disease with known neuropathology and clearly established boundaries, and a good example of a psychiatric disease that follows the medical model, which is the ideal that Kraepelin proposed for psychiatric diseases one hundred years ago (32). The problem is that 20 years of intensive research using the disease mechanism has led to many strategies targeting different mechanisms but, unfortunately, the outcome has been disappointing $(33,34)$. Thus, the attempts of neuroscience to use disease mechanisms to develop new treatments for Alzheimer's are not working well; a personalized treatment for Alzheimer disease would appear to be a fantasy due to the complexity of brain 
mechanisms. Therefore, even if we assume that Alzheimer disease is a psychiatric disease, the lack of success of the mechanistic disease approach to it is not good news for psychiatry. Alzheimer disease has 100 years of replicated neuropathological findings and 20 years of mechanistic studies based on reasonable understanding of its genetics. If one compares that with schizophrenia, for which Alzheimer completely failed as a neuropathologist, we find 100 years of arguments about schizophrenia's boundaries (30) and 60 years of treatment with drugs discovered by serendipity, which are considered not to be specific treatment for schizophrenia but are for all kinds of psychoses. In summary, even if major breakthroughs and ways of validating schizophrenia diagnosis occur in coming decades, our experience with Alzheimer disease tells us that our limited understanding of brain mechanisms is a major problem when compared with the highly developed mechanistic discipline of oncology.

\section{A forgotten way of personalizing treatment in psychiatry using clinical characteristics}

The author has proposed that consideration of disease mechanisms may not currently be a viable way of personalizing psychiatric treatment. However, past attempts to personalize psychiatric treatments based on the clinical profile of the patient remain ignored by current psychiatric researchers.

The idea behind these ignored approaches is that diseases, such as schizophrenia and depression, are not diseases but syndromes that can be carved out by sophisticated use of clinical symptoms in more specific diseases that can be better related to treatment response. These approaches were heavily invested in descriptive psychopathology, which was supposed to be the "language of psychiatry" (35) but interest in descriptive psychopathology has been decimated by the changes in US psychiatry since the DSM-III $(29,36)$. 
Ban has been one of the main traditional psychopharmacologists defending the concept that a disease's clinical profile can be used to group patients according to response $(37,38)$. $\mathrm{He}$ has focused on depression and schizophrenia but for space reasons, this editorial will focus only on schizophrenia. The current concept of schizophrenia presented in US nosological systems since the DSM-III combines the ideas of Kraepelin, Bleuler and Schneider $(30,39)$, but there is another model of schizophrenia that is, for the most part, ignored in US psychiatric textbooks and journals. As far as the author can tell, this alternative approach to diagnosing schizophrenia has rarely been described in US journals; two were selected as representative: Journal of Clinical Psychopharmacology and American Journal of Psychiatry. On 3/27/14, a PubMed search produced 4624 articles from the Journal of Clinical Psychopharmacology, of which 873 were on schizophrenia but none of them used this classification (adding the word "Leonhard" to the prior schizophrenia search provided no articles). On 3/27/14, a PubMed search produced 23,589 articles from the American Journal of Psychiatry, of which 3731 were on schizophrenia but no US articles used this classification. Only two articles (one letter to the editor and one twin genetic study) by the same German research group were identified.

Wernicke, one of the Kraepelin's main competitors, developed an alternative way of classifying psychosis. This approach to psychosis is sometimes labeled the approach of the Berlin school of psychiatry, since it was developed in Berlin. Wernicke trained Kleist, who trained Leonhard, who presented the final version of this alternative model of classifying psychosis. The Leonhard's psychosis model has been totally eclipsed by the Kraepelinian model. Wernicke died at a relatively early age in an accident (32). Most importantly, Leonhard worked at a Berlin hospital that stayed on the Russian side of Berlin and his textbook was not translated into English until recently (40). Leonhard was a very sophisticated clinician who did family and 
twin studies in hundreds of patients. His two major accomplishments include: 1) his proposal for the first time that familial history separates mono and bipolar depression, and 2) his theory that a group of psychoses lie between schizophrenia and manic-depressive illness; he called them cycloid psychoses (40). Leonhard proposed that schizophrenia is a syndrome including several illnesses (40), some of which are genetic and others of which are not. Systematic schizophrenia consists of non-genetic illnesses including paraphrenias, hebephrenias and catatonias. Unsystematic schizophrenias are genetic illnesses including cataphasia, affect-laden paraphrenia and periodic catatonia. Astrup (41), a Norwegian psychiatrist, conducted an important long-term outcome study in schizophrenia patients. He initially studied 189 patients by applying Leonhard's classification. Fish (42) reclassified, with Astrup, another 285 chronic schizophrenia patients. Finally, Fish included all 474 patients with schizophrenia in an article which reported that $<1 / 4$ of systematic schizophrenia patients versus $>4 / 5$ of unsystematic schizophrenia patients responded to first-generation antipsychotics (42). Later on, Guy et al. (43) in an international survey of 768 patients with chronic schizophrenia, found that tardive dyskinesia was overrepresented in patients with systematic versus those with unsystematic schizophrenia (13.3\% vs $4.3 \%$ ). In a small group of 50 patients with chronic schizophrenia in Germany, Beckmann et al. (44) proposed that the prognosis did not appear to change with antipsychotics when compared with Leonhard's observations. All of these outcome studies were conducted by sophisticated clinicians who did not use blinding or placebo in their studies but suggested that systematic schizophrenia does not respond well at least to first-generation antipsychotics. There is little reason to think that these clinical researchers were biased toward finding greater response to antipsychotics in unsystematic schizophrenia, since Leonhard developed his classification in the pre-neuroleptic era. 
The author, along with Ban $(37,38)$, believes that schizophrenia and major depressive disorder are syndromes rather than illnesses, but all of these pre-DSM-III attempts to divide them into more homogenous subgroups that may predict treatment response remain forgotten. They are not considered because they were conducted by sophisticated clinicians who did not use wellcontrolled designs with blinding and placebo. Due to these methodological weaknesses, these studies remain forgotten by current psychopharmacologists who only value clinical trials using well-controlled designs. Contemporary psychopharmacologists may have forgotten that psychopharmacological drugs were discovered by sophisticated clinicians without using wellcontrolled designs. The use of well-controlled designs has not brought any revolutionary changes in psychopharmacology, just some second-generations drugs with possibly some better ADR profiles but of doubtful greater efficacy. The author thinks that it will be interesting to incorporate some of these attempts to subdivide schizophrenia and major depression in future well-controlled pragmatic studies of psychotropic drugs. Unfortunately, this will require intensive clinical training of psychiatrists involved in the diagnosis and assessment of patients. When psychiatrists are heavily invested in using biological mechanisms to personalize psychiatric treatments, it may be too late to try to go back to forgotten attempts to personalize treatment according to sophisticated clinical assessments.

\section{Conclusions}

Personalized medicine has finally been discussed in psychiatric journals but psychiatrists have mainly focused on the promise of using disease mechanisms to personalize treatment. Psychiatric disorders such as schizophrenia and depression are not diseases, in the medical sense, and are probably more like syndromes. Moreover, even if one focuses on Alzheimer disease, which is definitively closer to the concept of brain disease, current mechanistic approaches have 
been rather disappointing, and it is not clear that personalized prescription for treating Alzheimer disease can take place in the next 1-2 decades.

Instead of spending much time and effort focusing on the mechanisms of diseases that may be syndromes rather than diseases, the author believes that psychiatrists should 1) learn more about personalizing prescription using the drug mechanisms that are common among syndromes, and 2) reconsider prior attempts to use sophisticated clinical approaches that try to subdivide psychiatric syndromes into groups that may be more homogenous for treatment response. First, learning more about personalized prescription as understood by pharmacologists may bring definitive but only modest improvements (12), either by using pharmacokinetic mechanisms to personalize drug treatment with a few psychiatric drugs, or by considering some pharmacodynamic mechanisms for personalizing drug selection for a few psychiatric drugs (clinicians can rule out some patients through the use of HLA testing before prescribing carbamazepine in East Asian patients). Second, increasing our knowledge of more sophisticated clinical approaches of classification of psychiatric syndromes and introducing them in clinical trials cannot be worse than the current situation in which pharmaceutical companies, after 3 or 4 decades, have failed to generate mechanistically new drugs and are abandoning psychiatry (45). It is possible that more clinically homogenous subgroups of patients may be suitable for the mechanistic approaches. For example, a genetic variant has been associated with one of Leonhard's diseases within the schizophrenia syndrome, periodic catatonia (46). 


\section{Acknowledgments}

The author acknowledges Lorraine Maw, M.A., at the Mental Health Research Center at Eastern State Hospital, Lexington, KY, who helped in editing this article. Cristoph Hiemke, Ph.D., used the phrase "each drug is an individual" in an e-mail to the author on 7/10/13 as he commented on one of the author's articles on clobazam therapeutic drug monitoring.

\section{Financial Support}

This article received no specific grant from any funding agency, commercial business, or not-for-profit institution.

\section{Statement of interest}

Dr. de Leon personally develops his presentations for lecturing, has never lectured using any pharmaceutical or pharmacogenetic company presentation, and has never been a consultant for pharmacogenetic or pharmaceutical companies. In the past, Dr. de Leon has received researcher-initiated grants from Eli Lilly (one ended in 2003 and the other, as co-investigator, ended in 2007) from Roche Molecular Systems, Inc. (ended in 2007), and in a collaboration with Genomas, Inc., from the NIH Small Business Innovation Research program (ended in 2010). He was on the advisory boards of Bristol-Myers Squibb (2003/04) and AstraZeneca (2003). Roche Molecular Systems supported one of his educational presentations, which was published in a peer-reviewed journal (2005). His lectures have been supported once by Sandoz (1997), twice by Lundbeck (1999 and 1999), twice by Pfizer (2001 and 2001), three times by Eli Lilly (2003, 2006, and 2006), twice by Janssen (2000 and 2006), once by Bristol-Myers Squibb (2006), and seven times by Roche Molecular Systems, Inc. (once in 2005 and six times in 2006).

\section{References}


1. OSBORN LA. Psychiatry and medicine: An introduction to personalized medicine. New York: McGraw Hill; 1952.

2. DE LEON J. Evidence-based medicine versus personalized medicine: are they enemies? J Clin Psychopharmacol 2012;32:153-164.

3. GARROD AE. Inborn errors of metabolism. London: Oxford University Press; 1909.

4. Vogel F. Moderne probleme der Humangenetik. Ergeb Inn Med Kinderheild $1959 ; 12: 52-125$.

5. KANIGEL R. Apprentice to genius: The making of a scientific dynasty. New York: Macmillan Publishing Company; 1986.

6. BRodie BB, Cosmides GJ. Toxicology and the biomedical sciences. Science 1965;148:1547-1554.

7. MeYer UA. Pharmacogenetics - five decades of therapeutic lessons from genetic diversity. Nat Rev Genet 2004;5:669-676.

8. FODOR SP. Massively parallel genomics. Science 1997;277:393-395.

9. SCIENCE. New research horizons. Science 1997;278:2039.

10. DE LEON J. The future (or lack of future) of personalized prescription in psychiatry. Pharmacol Res 2009;59:81-89.

11. Hiemke C, Baumann P, Bergemann N, ET AL. AGNP consensus guidelines for therapeutic drug monitoring in psychiatry: update 2011. Pharmacopsychiatry 2011;44:195-235.

12. CRettol S, de LeOn J, HiemKe C, EAP C. Pharmacogenomics in psychiatry: from therapeutic drug monitoring to genomic medicine. Clin Pharmacol Ther 2014;95:254257. 
13. DE LEON J, SPINA E, DiAZ FJ. Clobazam therapeutic drug monitoring: a comprehensive review of the literature with proposals to improve future studies. Ther Drug Monitor $2013 ; 35: 30-47$.

14. WAGNER JA. Overview of biomarkers and surrogate endpoints in drug development. Dis Markers 2002;18:41-46.

15. Dienstmann R, RodOn J, TABERnERo J. Drug development in the era of personalized oncology: From population-based trials to enrichment and prescreening strategies. Am Soc Clin Oncol Educ Book 2012;32:168-172.

16. WALDROP M. Complexity: The emerging science at the edge of order and chaos. New York: Touchstone Books; 1992.

17. BARABÁsi AL, GulbAhCE N, Loscalzo J. Network medicine: a network-based approach to human disease. Nat Rev Genet 2011;12:56-68.

18. MCGORRY PD. Early clinical phenotypes, clinical staging, and strategic biomarker research: building blocks for personalized psychiatry. Biol Psychiatry 2013;74:394-395.

19. WeISSMAN MM, BROWN AS, TALATI A. Translational epidemiology in psychiatry: linking population to clinical and basic sciences. Arch Gen Psychiatry 2011;68:600-608.

20. Wong EH, Fox JC, NG MY, ET AL. Toward personalized medicine in the neuropsychiatric field. Int Rev Neurobiol 2011;101:329-349.

21. HECKERS S. Future in psychopathology research. Schizophr Bull 2014;40 suppl 2:S147S151.

22. Ozomaro U, Wahlestedt C, Nemeroff CB. Personalized medicine in psychiatry: problems and promises. BMC Med 2013;11:132. 
23. DE LEON J, ARMSTRONG SC, CozZA KL. Clinical guidelines for psychiatrists for the use of pharmacogenetic testing for CYP450 2D6 and CYP450 2C19. Psychosomatics 2006;47:75-85.

24. ARRANZ MJ, DE LEON J. Pharmacogenetics and pharmacogenomics of schizophrenia: a review of last decade of research. Mol Psychiatry 2007;12:707-747.

25. INSEL TR, SCOLNICK EM. Cure therapeutics and strategic prevention: raising the bar for mental health research. Mol Psychiatry 2006;11:11-17.

26. KAPUR S, PhiLlips AG, INSEL TR. Why has it taken so long for biological psychiatry to develop clinical tests and what to do about it? Mol Psychiatry 2012;17:1174-1179.

27. MCHUGH PR. Striving for coherence: psychiatry's efforts over classification. JAMA 2005;293:2526-2528.

28. LAIN EnTRALGo P. Historia de la medicina. Barcelona: Salvat; 1978.

29. DE LEON J. Is psychiatry scientific? A letter to a 21 st century psychiatry resident. Psychiatry Investig 2013;10:205-217.

30. DE LEON J. One hundred years of limited impact of Jaspers' General Psychiatry in the US psychiatry. J Nerv Ment Dis 2014;202:79-87.

31. BERRIOS GE. Alzheimer's disease: a conceptual history. Int J Geriatr Psychiatry 1990;5:355-365.

32. DE LEON J. DSM-5 and Research Domain Criteria: One hundred years after Jaspers's General Psychopathology. Am J Psychiatry 2014 May.

33. Morley JE. Alzheimer's disease: future treatments. J Am Med Dir Assoc 2011;12:1-7.

34. Crenshaw DG, GotTschalk WK, Lutz MW, et AL. Using genetics to enable studies on the prevention of Alzheimer's disease. Clin Pharmacol Ther 2013;93:177-185. 
35. BAN TA. Neuropsychopharmacology and the forgotten language of psychiatry. International Network for the History of Neuropsychopharmacology (INHN) E-Book, 2013. http://inhn.org/previews/neuropsychopharmacology-and-the-forgotten-languageof-psychiatry.html

36. ANDREASEN NC. DSM and the death of phenomenology in America: an example of unintended consequences. Schizophr Bull 2007;33:108-112.

37. BAN TA. Prolegomenon to the clinical prerequisite: psychopharmacology and the classification of mental disorders. Prog Neuropsychopharmacol Biol Psychiatry 1987;11:527-580.

38. BAN TA. Towards a clinical methodology for neuropsychopharmacological research. Neuropsychopharmacol Hung 2007;9:81-90.

39. HoENIG J. The concept of schizophrenia: Kraepelin - Bleuler - Schneider. Br J Psychiatry 1983;142:547-556.

40. LEONHARD K. Classification of endogenous psychoses, $5^{\text {th }}$ ed. New York: Irvington Publisher; 1979.

41. Astrup C. Schizophrenia: Conditional reflex studies. Springfield, IL: Thomas; 1962.

42. FISH F. The influence of the tranquillisers on the Leonhard schizophrenic syndromes. Encéphale 1964;53:245-249.

43. GUY W, BAN TA, WILSON WH. An international survey of tardive dyskinesia. Prog Neuropsychopharmacol Biol Psychiatry 1985;9:401-405.

44. BeCKMANn H, FritZE J, FranzeK E. The influence of neuroleptics on specific syndromes and symptoms in schizophrenics with unfavourable long-term course. A 5-year follow-up study of 50 chronic schizophrenics. Neuropsychobiology 1992;26:50-58. 
45. FIBIGER HC. Psychiatry, the pharmaceutical industry, and the road to better therapeutics. Schizophr Bull 2012;38:649-650.

46. Stöber G, Kohlmann B, IEKIERA M, ET AL. Systematic mutation analysis of KIAA0767 and KIAA1646 in chromosome 22q-linked periodic catatonia. $B M C$ Psychiatry.2005;5:36. 